\title{
INSTITUCIONES Y FETICHES: EL PROBLEMA DE LA "MATERIA OSCURA" EN LAS CIENCIAS SOCIALES ${ }^{1}$
}

\author{
INSTITUTIONS AND FETISHES: THE "DARK MATTER" PROBLEM IN SOCIAL SCIENCES \\ INSTITUIÇÕES E FETICHE: O PROBLEMA DA "MATÉRIA ESCURA" NAS CIÊNCIAS \\ SOCIAIS
}

\section{Henry Mora Jiménez²}

\begin{abstract}
Resumen
El campo de las ciencias sociales que estudia los conceptos asociados a entidades colectivas se ha topado desde sus inicios con el problema de la visibilidad de lo invisible. Es la teoría del fetichismo. Puede tratarse de colectivos parciales (empresas, escuelas, ejércitos), o de colectivos generales, como la división social del trabajo, el mercado y el Estado. Se trata de un mundo de sujetos fantasmales que nos pueden dominar y que en gran medida llegan a dominarnos. Si no captamos y entendemos el significado de estos fetiches, y dado el creciente papel de las instituciones desde los albores de la civilización hasta nuestros días, vis a vis la pronunciada reducción de las relaciones humanas directas en las sociedades modernas, comprenderemos muy poco del universo social y su fetichización. Por eso decimos que estos fetiches son, en las ciencias sociales, el equivalente a la "materia oscura" de la astrofísica.

Palabras claves: institucionalización; relaciones humanas; fetiches; materia oscura; libertad
\end{abstract}

Doi: https://doi.org/10.15359/eys.24-55.2

Fecha de recepción: 07-15-2019. Fechas de reenvíos: 16-01-2019, 24-01-2019. Aceptado el 31-01-2019. Publicado el 06-02-2019.

${ }^{1}$ El presente artículo es una versión revisada y ampliada de "Sobre el desdoblamiento del mundo en las ciencias sociales", publicado en la Revista Paquidermo en enero de $\underline{2013}$, escrito por Franz J. Hinkelammert y Henry Mora Jiménez.

2 Doctor en Economía, catedrático e investigador en Observatorio Económico y Social (OES-UNA) de la Escuela de Economía, Universidad Nacional, Costa Rica. Correo electrónico: hmoraj@gmail.com.

126

Henry Mora Jiménez

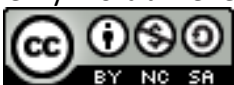

Revista Economía y Sociedad by Universidad Nacional is licensed under a CreativeCommons Reconocimiento-NoComercial- 


\begin{abstract}
The field of social sciences that studies concepts associated with collective entities, that is the theory of fetishism, has faced the problem of the visibility of the invisible since the beginning. It may include partial collectives (companies, schools, armies) or general collectives, such as the social division of labor, market, and State. It is a world of ghostly subjects that have the possibility to dominate us and that ultimately, and to a great extent, end up doing so. Given the growing role of institutions from the dawn of civilization to the present day regarding the pronounced reduction of direct human relationships in modern societies, if we do not grasp and understand the meaning of these fetishes, we will understand very little of the social universe and its fetishization. This is the reason why we state that these fetishes in social sciences are the equivalent of "dark matter" in astrophysics.
\end{abstract}

Keywords: institutionalization; human relations; fetishes; dark matter; freedom

\title{
Resumo
}

O campo das ciências sociais que estuda os conceitos associados às entidades coletivas tem enfrentado desde o início o problema da visibilidade do invisível. É a teoria do fetichismo. Podem se tratar de coletivos parciais (empresas, escolas, exércitos) ou coletivos gerais, como a divisão social do trabalho, o mercado e o Estado. Trata-se de um mundo de sujeitos fantasmagóricos que podem nos dominar e que em grande parte chegam a nos dominam. Se não captarmos e compreendermos o significado desses fetiches e, dado o crescente papel das instituições desde os primórdios da civilização até os dias atuais, diante da redução pronunciada das relações humanas diretas nas sociedades modernas, entenderemos muito pouco do universo social e sua fetichização. É por isso que dizemos que esses fetiches são, nas ciências sociais, o equivalente à "matéria escura" da astrofísica.

Palavras-chave: Institucionalização; relações humanas; fetiches; matéria escura; liberdade 
Desde hace más de 40 años, los astrofísicos y cosmólogos se desvelan por entender la materia oscura del universo ${ }^{3}$. Pues bien, en economía crítica, la tradición iniciada por Marx (y continuada entre otros, por Hinkelammert), se planteó un problema similar para lo que hoy llamamos "ciencias sociales", y eso ya hace 150 años. Es el problema de los colectivos invisibles, cuya existencia los seres humanos percibimos, pero no directamente: ¿Quién ha visto y tocado una corporación, un sistema educativo, un Estado o un régimen de propiedad?

Lo que naturalmente sí vemos son los elementos materiales de estas instituciones: edificios, bibliotecas, funcionarios públicos que llevan a cabo ciertas tareas, títulos de propiedad o cercas que demarcan el perímetro de una hacienda ganadera. Pero el concepto clave relativo a estos colectivos y que queremos resaltar no se refiere a estos elementos tangibles, sino al conjunto o a la totalidad de sus actividades. Como tales, se refieren a realidades invisibles. No los vemos directamente, pero los percibimos y tenemos vivencia de ellos. A estos colectivos los denominamos instituciones, y pueden transformarse en fetiches, esto es, creaciones humanas que llegan a dominar a quienes las crearon. $Y$ aunque no los vemos directamente, condicionan la vida de todas las personas, por lo que, de manera similar a la materia oscura de la física, una multiplicidad de fenómenos, en este caso sociales, no los podríamos entender a menos que estos mismos puedan incluirse en nuestras teorías sobre la sociedad ${ }^{4}$. Como colectivos o instituciones son totalidades, y el ojo humano no puede ver totalidades, aunque su vivencia sí pueda percibirlas. El ojo humano solo puede ver personas u objetos (dentro de su ángulo de visión), pero ni siquiera todas las personas o todos los objetos, sino solamente aquellos que están al alcance de la vista. Pero resulta claro que los condicionamientos de nuestra vida incluyen, en última instancia, todas las personas y entidades existentes, aunque no nos resulten visibles. Algo similar sucede con las instituciones, esas objetivaciones sensorialmente no perceptibles de las relaciones humanas.

Pues bien, el campo de las ciencias sociales que estudia los conceptos de colectivos, la visibilidad de lo invisible, es la teoría del fetichismo. Puede tratarse de colectivos parciales (empresas, escuelas, ejércitos), o de colectivos generales, como la división social del trabajo, en relación con la cual debemos estudiar la conformación de las relaciones de producción y el surgimiento del Estado. La división social del trabajo es, de hecho, el condicionamiento fundamental, pues se

\footnotetext{
3 "Materia Oscura": este es el nombre con el que se ha designado a todo aquello que los astrónomos no pueden ver ni detectar en forma directa, pero que se hace evidente a través de su atracción gravitatoria sobre otros cuerpos celestes. Identificar a esta entidad omnipresente y misteriosa que se hace sentir en todas partes del Universo se ha convertido en un desafío para la astrofísica moderna (Baker, 2009, pp. 249-253).

${ }^{4}$ En el caso de la física, nos referimos a fenómenos como las lentes gravitacionales, la velocidad de rotación de las galaxias o la misma existencia de estas. En el caso de las ciencias sociales, nos referimos principalmente a las macro institucionalidades del mercado y el Estado, de las que "no es posible conocer ningún fundador, porque no aparecen por un acto de fundación [a diferencia de una institución parcial como una empresa]. Fundando 'intencionalmente' instituciones parciales, se fundan y se reformulan de una manera 'no-intencional' el mercado y el Estado, la sociedad civil y la sociedad política" (Hinkelammert, 2002, p. 45).
} 128 
refiere no solo a la asignación o distribución de las distintas actividades humanas, sino también a las mismas condiciones materiales de la vida; deciden, por tanto, sobre la vida o la muerte de las personas que conviven en interrelación e interdependencia. Si la posibilidad de vivir es el problema básico del ser humano y del ejercicio de su libertad, la división social del trabajo se convierte en la referencia clave para el estudio de las instituciones en su totalidad.

Tenemos, además, las dos instituciones centrales, sin las cuales es imposible entender nuestras sociedades actuales: el mercado y el Estado. Ninguna de ellas es institución parcial, sino que ambas abarcan y envuelven al conjunto de todas las instituciones parciales. A ciencia cierta son "institucionalidades" y no simples "instituciones parciales", porque confieren los criterios de organización del conjunto de las instituciones parciales. Por eso están en todas partes, aunque no las vemos directamente, justo como a la "materia oscura" del universo.

\section{Sobre el desdoblamiento de la realidad y su significación para las ciencias sociales}

Veamos una sencilla aplicación de la teoría del fetichismo de Marx partiendo del concepto de la empresa capitalista, para examinar el desdoblamiento de la realidad a partir de la institucionalización de las relaciones humanas en su forma social mercantil ${ }^{5}$.

La mayoría de los idiomas establecen la diferencia entre una "fábrica" y una "empresa”. La fábrica es, estrictamente hablando, la única experiencia sensorial que podemos tener de una empresa. Se trata de una experiencia sensitiva y visible. La fábrica es un agregado de edificios, máquinas, materias primas, productos terminados y seres humanos. En ella se producen mercancías, que también son visibles y pueden tener tamaños, formas, texturas, sabores y sonidos diferenciados. Pero las fábricas no son las empresas, y tanto es así que en el balance general de una empresa (activo, pasivo y patrimonio), la fábrica está en el lado de sus activos, mientras la empresa es, podemos decir, "todo el balance de situación". Como empresa ella es invisible, pues de hecho se trata de una ficción jurídica. Por eso podemos indicar una fábrica señalándola con nuestro dedo índice o tocar cualquier parte de esta con nuestras propias manos. Sin embargo, una empresa, en el sentido empírico, no es aprehensible de esta manera, no la podemos señalar ni tocar. Además, una empresa puede estar ubicada físicamente en un país cualquiera, pero "domiciliada" en un paraíso fiscal. La empresa, como todo orden institucionalizado, se encuentra fuera del mundo empírico.

Tampoco podemos ver "el mercado", a pesar de que muchas veces tenemos una idea de dónde encontrar un mercado particular. Lo que sí podemos llegar a ver son las personas que compran, venden o intercambian productos, siempre que lo hagan en un determinado espacio físico. Tampoco podemos ver un ejército (el conjunto de las fuerzas armadas de una nación), aunque

5 Aunque la categoría marxiana de fetichismo tiende a asociarse a la forma mercantil, Hinkelammert la ha interpretado como un fenómeno inherente a la condición humana (Hinkelammert, 1981). 
podemos ver personas uniformadas y armadas que golpean a manifestantes en una protesta popular. Sabemos que eso es una acción del ejército que a su vez es un "cuerpo" del Estado, pero este hecho no lo podemos derivar categóricamente de ninguna experiencia empírica: no podemos asegurar que alguien sea policía o soldado por el solo hecho de que lleve un uniforme. Puede ser un impostor o incluso un ladrón disfrazado de policía. Tampoco el dinero se puede experimentar empíricamente, ya que de un signo de dinero no se puede concluir que se trata efectivamente de dinero de curso legal: podría haber perdido su validez, o podría ser falsificado $y$, por tanto, un simple pedazo de papel. Lo mismo vale para escuelas, iglesias, sistemas educativos, sistemas de salud, etc. No tenemos ninguna experiencia empírica del orden institucionalizado, sino solamente de los elementos empíricos materiales en los cuales descansa su existencia. Pero sin su entendimiento -y dado el creciente papel de las instituciones desde los albores de la civilización, vis a vis la pronunciada reducción de las relaciones humanas directas en las sociedades modernas-, comprenderíamos muy poco del universo social ${ }^{6}$.

Detrás de todas las condiciones materiales empíricamente experimentables de estas instituciones existen cuasi sujetos, que son las instituciones mismas. Detrás del edificio de una escuela está el sujeto escuela como institución, detrás de la fábrica está el sujeto empresa, detrás del cuartel un ejército, detrás de la Casa Blanca un Estado. Se trata de un mundo de sujetos fantasmales que nos pueden dominar y que, en gran medida, Ilegan a hacerlo. Porque si resulta cierto (como creemos), que la abolición de todo orden institucionalizado (la anarquía) es empíricamente imposible, entonces también tiene que ser cierto que las condiciones de existencia de este mundo fantasmal son formas inevitables de la convivencia humana, casi tan antiguas como la humanidad misma. Por tanto, de la inevitabilidad de la existencia del mundo institucional se pueden derivar, analíticamente, las normas de convivencia correspondientes, que resultan ser más o menos obligatorias, a menos que estemos dispuestos a precipitarnos en el caos o, incluso, acabar en el suicidio colectivo.

Por eso, en las ciencias sociales necesitamos conceptos de lo material, de la experiencia y de la "objetividad" que vayan más allá de lo empíricamente visible y experimentable. Los espectros institucionales no se pueden experimentar con los sentidos, pero si queremos que las ciencias sociales tengan sentido, tienen que ser asumidos como parte del mundo que experimentamos.

Resulta que, como en el caso de la empresa capitalista, estos fantasmas tienen balances de situación, presupuestos anuales e incluso personería jurídica. Se les puede ofender o acusar, se les puede estafar, se puede ablandar sus corazones con sollozos y hasta pueden envían mensajes y correspondencia. Cuando alguna empresa cualquiera nos remite en diciembre una tarjeta con saludos navideños, estas tarjetas llevan la firma litografiada de alguna persona "en nombre de la empresa", o simplemente, el logotipo de esta. De esta manera deducimos que tal empresa tiene

${ }^{6}$ Ocurre como con la materia normal vs la materia oscura: se calcula que la primera solo representa un veinte por ciento de la segunda y apenas un cuatro por ciento de todo lo que existe en el universo. 130

Henry Mora Jiménez



Revista Economía y Sociedad by Universidad Nacional is licensed under a CreativeCommons Reconocimiento-NoComercial- 
una voluntad que nos hace presente; pero la orden de enviar la tarjeta no viene siquiera de la asamblea de accionistas, sino de "la empresa", porque también la asamblea de accionistas actúa "en nombre de la empresa", que es un ente invisible y fantasmal.

Pero estos fantasmas son, por supuesto, productos humanos, aunque en algunos casos nadie los haya producido intencionalmente. Son inteligibles -al menos en la tradición iniciada por Marxcomo productos no intencionales o indirectos de la acción humana directa. Pero más aún, el ser humano no puede dejar de producirlos. Llegan a ser parte del mundo experimentado, aunque no los podamos experimentar por medio de los sentidos; los experimentamos por medio de un comunicado de un Juzgado civil o por la cachiporra de un policía?.

De esta manera resulta que el mundo experimentado por los seres humanos y que las ciencias sociales intentan explicar es un mundo desdoblado. En primer lugar, se trata del mundo empírico de las condiciones materiales de la posibilidad de los proyectos humanos y, en segundo lugar, de un mundo cuasi empírico que resulta de los efectos no-intencionales de la acción humana intencional y cuya existencia (como en el caso de la materia oscura en astrofísica) hay que concluirla, porque se trata de un mundo invisible: es el mundo de las formas sociales y de las objetivaciones institucionales de la subjetividad humana ${ }^{8}$.

\section{Fetiches y máscaras}

En el campo económico tenemos un claro ejemplo de esta existencia desdoblada del mundo empírico, tal como ya lo hemos indicado al advertir la diferencia entre fábrica y empresa. El mundo empírico u objetivo de la experiencia humana que estudia la ciencia social está sometido a leyes de la necesidad, mientras que el mundo cuasi empírico está sometido a leyes de la inevitabilidad. En un sentido estricto, este mundo cuasi empírico es un mundo sobrenatural, metafísico; aunque eso no signifique que no se lo pueda experimentar. Aparecen también pensamientos que pretenden la abolición de este mundo sobrenatural, aunque estas aboliciones resulten imposibles.

\footnotetext{
7 El Ilamado racionalismo crítico (Karl Popper) asumió una explicación análoga de las instituciones, pero no logró derivar de ella una teoría del orden institucional, es decir, del orden del mercado, la propiedad y el Estado (Hinkelammert, 2002).

8 "Marx desarrolla su concepto de la subjetividad del mundo objetivo en su primera tesis sobre Feuerbach.... Se trata por supuesto, de la actuación de cada uno que tiene que ser práctico-crítica... Esta subjetividad Marx la vio como objetivamente dada. Tomar el objeto simplemente como objeto dado, es reducción de la objetividad y no corresponde a la objetividad, que es subjetiva. Que el objeto es subjetivo, para Marx es una verdad objetiva. No se trata de la relación sujeto-objeto de Descartes, de Kant o de Hegel; es la praxis. El objeto tiene siempre la dimensión de la praxis y es, como tal, subjetivo" (Hinkelammert, 2012, p. 197).
} 
La derivación de la existencia de este mundo sobrenatural tiene muchos caminos. Una corporación como The Coca-Cola Company ${ }^{9}$ no puede sonreír, porque es una ficción jurídica, sin embargo, un empresario sí puede hacerlo y eso es visible empíricamente. En muchas ocasiones se puede concluir que, a través de la risa del empresario, sonríe la propia corporación. Si esta goza de "buena salud" porque las ganancias son boyantes, entonces sonríe, pero lo puede hacer solamente a través de la risa del empresario quien la representa en este mundo. Es lo que llama Marx la "máscara característica del capital".

Pero la prosperidad no es necesariamente para todos los sujetos, y otros pueden llorar. Si un obrero de la corporación es despedido "por justa causa" (por un bajón en las ventas o porque se ha sindicalizado), la empresa no asume ninguna responsabilidad, porque «el empresario es a la empresa, lo que la empresa es al mercado». El mercado es el que ha decidido que la empresa debe despedir al obrero (aunque en ocasiones esto sea una farsa, como en el caso de obreras y obreros sindicalizados). Aunque el mercado sea también un ente fantasmal, realiza; sin embargo, tales decisiones. "¿Y qué puede hacerse, si la sentencia del mercado no se puede cambiar a voluntad ni contravenir sin causar males mayores?" Ciertamente es algo de lamentar, pero es inevitable. Por eso, quizás ahora no sonría la empresa, pero su personal despedido y sus familias llorarán, sin presentar ninguna máscara característica ${ }^{10}$.

\section{Institucionalización y libertad}

Existe, por tanto, un mundo de experiencia que es metafísico, que incluso dicta normas a nuestro mundo empírico y lo domina. Es la ley como la cárcel del cuerpo, son los "dioses terrestres": el dinero, el mercado, el capital, el Estado; aunque no hayan surgido necesariamente con esa "marca"11.

Pero como ya se ha afirmado, la no-institucionalización de las relaciones humanas es imposible, no la podemos abolir, hagamos lo que hagamos, siempre regresa. Por eso la pregunta puede ser solamente, ¿cómo y hasta qué punto se puede aprisionar este fantasma?

\footnotetext{
${ }^{9}$ Una historia semejante sobre Peugeot S.A. es contada por Yuval Noah Harari (2018) en De animales a dioses, pp. 38-46, "La leyenda de Peugeot".

${ }^{10}$ Si un movimiento de madres jóvenes aboga por extender el período de lactancia de 3 a 6 meses, las cámaras empresariales dirán que no objetan del todo el cambio en la ley vigente, pero advierten que menos mujeres serán contratadas en el sector privado, pues las empresas tendrán que apechugar con parte del financiamiento de los tres meses adicionales de lactancia. Las empresas quizás no sonrían, pero el mercado manda y los bebés lactantes llorarán.

${ }^{11}$ Marx cita el Apocalipsis al denunciar el dinero como dios terrestre: "Estos tienen un consejo, y darán su potencia y autoridad a la bestia. Y que ninguno pudiese comprar o vender, sino el que tuviera la señal o el nombre de la bestia, o el número de su nombre." (citado en Hinkelammert y Mora, 2014, pp. 171-172). Se podría añadir la siguiente cita: "...la tierra entera siguió maravillada a la Bestia. Y se postraron ante el Dragón, porque había dado el poderío a la bestia, y se postraron ante la bestia diciendo: ‘¿Quién como la Bestia?, ¿Y quién puede luchar contra ella?'” (Ap. 13:34).
}

132

Henry Mora Jiménez

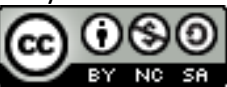

Revista Economía y Sociedad by Universidad Nacional is licensed under a CreativeCommons Reconocimiento-NoComercial- 
La respuesta de Marx es su "criterio de verdad" sobre el orden institucional: ¿hasta qué grado se trata al ser humano como el ser supremo para el ser humano? Y hasta cuánto eso se logre o no depende de hasta qué grado se trata al ser humano como "un ser humillado, sojuzgado, abandonado y despreciable". Este es el criterio para determinar hasta qué grado las instituciones son "dioses falsos", fetiches. Cuanto más siguen su propia lógica y las fuerzas compulsivas de los hechos, tanto más falsos son y tanto más escapan a nuestro control.

\section{Referencias}

Baker, J. (2009). 50 cosas que hay que saber sobre física. Ariel. Recuperado de www.librosmaravillosos.com

Hararri, Y. N. (2018). De animales a dioses. Breve historia de la humanidad. México: Penguin Random House Grupo Editorial.

Hinkelammert, F. (1981). Las armas ideológicas de la muerte (2 ${ }^{\text {da }}$ edición revisada y ampliada). San José, Costa Rica: DEl.

Hinkelammert, F. (2002). Crítica de la razón utópica (Edición ampliada y aumentada). Bilbao: Editorial Desclée.

Hinkelammert, F. (2012). Raíces del pensamiento crítico. Universidad Distrital Francisco José de Caldas, Colombia.

Hinkelammert, F. y Mora, J. (2013). Sobre el desdoblamiento del mundo en las ciencias sociales. Revista Paquidermo. Recuperado de https://revistapaquidermo.com/archives/7730

Hinkelammert, F. y Mora, J. (2014). Economía, vida humana y bien común. San José, Costa Rica: Editorial Arlekín. 